\title{
Interfacing Microfluidics and Laser Desorption/Ionization Mass Spectrometry by Continuous Deposition for Application in Single Cell Analysis
}

\author{
Andrea Amantonico $\S^{\star}$, Pawel L. Urban, Joo Yeon Oh, and Renato Zenobi \\ §SCS Poster Prize Winner
}

\begin{abstract}
We present a simple method for continuous deposition of effluent originating from a microfluidic device on a flat metal surface for subsequent analysis by matrix-assisted laser desorption/ionization mass spectrometry (MALDI-MS). The sample is delivered using a microscale fused silica capillary and passed onto the surface of a stainless steel plate coated with a layer of a standard matrix. The key parameters optimized in order to obtain high quality and reproducible sample traces are: i) sample flow rate, ii) speed of the XY-stage movement, and iii) distance of the capillary tip from the plate. Tapering the capillary end as well as surface functionalization to induce hydrophobicity were shown to further enhance the deposition process. The described continuous deposition method is compared with a previously published mass spectrometric method utilizing a piezoelectric microdispenser for microspotting onto the MALDI plates which enabled detection of primary metabolites at the single cell level. Research is underway to adapt the continuous deposition as an interface for single cell metabolite detection and enhancement of quantitative abilities of the MALDI methodology. We envisage that the presented continuous deposition method may also be suitable for sensitive detection of analytes using other surface analysis tools.
\end{abstract}

Keywords: Hyphenated techniques · Mass spectrometry · Microfluidics · Microscale sample preparation · Single cell analysis · Writing on MALDI plates

\section{Introduction}

Mass spectrometry (MS) is a unique analytical technique that can cope with the chemical diversity of analytes in biological samples. It has already become a key technique in systems biology and metabolomics. ${ }^{[1,2]}$ Matrix-assisted laser desorption/ ionization (MALDI) mass spectrometry is a particularly advantageous platform with numerous applications for sensitive detection of biomolecules. ${ }^{[3]} \mathrm{As}$ it is in principle an off-line technique for analyzing samples deposited on flat surface, it is frequently

\footnotetext{
${ }^{*}$ Correspondence: A. Amantonico

ETH Zurich

Department of Chemistry and Applied Biosciences $\mathrm{HCl}$ D 330

Wolfgang-Pauli-Str. 10

$\mathrm{CH}-8093$ Zürich

Tel: + 41-44-632-4783

Fax: +41-44-632-1292

E-mail: amantonico@org.chem.ethz.ch
}

utilized in the analysis of discrete fractions collected downstream a separation step.

Although several approaches to on-line coupling of continuous flow sample treatment techniques to MALDI can be found in the literature, ${ }^{[4-6]}$ these set-ups have not become widespread, probably due to the technical difficulties associated with stability of such on-line vacuum interfaces for MALDI. Therefore, it is clear that due to spatial and temporal separation of the sample deposition and analyte ionization steps, MALDI is more successfully run in off-line detection mode.[6-10] The 'off-line' nature of MALDI should not be perceived as a disadvantage though. Off-line coupling of sample treatment steps with MALDI can in fact bring advantages normally absent in on-line hyphenated techniques, for example:

- Two or more steps of the analytical routine can be separated in time and space, hence none of the instruments involved in the process is blocked due to prolonged optimization or troubleshooting of one of the steps (e.g. separation and detection).

- Archiving of arrays of samples is possible.

- Samples can be reanalyzed using different instruments; several instruments/ detectors can be used sequentially, so as to fulfill particular analytical requirements.

- No dilution is caused due to mixing with sheath liquid; matrix can be deposited on the plate prior to the sample.

- There is the possibility for sample treatment on plate ('lab-on-plate'); for example, analytes can be concentrated, extracted or separated directly on the plate.

- MALDI instruments can be used as they are, whilst on-line coupling normally requires considerable modifications to the ion source module.

A previous study conducted in our group demonstrated that spotting extremely small volumes of samples $(<500 \mathrm{pl})$ on a small surface area enables detection of a wide set of endogenous metabolites with a sensitivity that allows for chemical analysis of amounts corresponding to single yeast cells $^{[11]}$ (Fig. 1): this employed a combination of microscale sample preparation, thin layer matrix deposition and negative ion mode detection by MALDI-MS. This analytical method is thought to be amenable to coupling with a microfluidics-based cell treatment (e.g. lysis) in order to handle the minute metabolite quantities originating from single cells. It has been pointed out 
that such a combination may become an enabling tool for systems biology since it will allow for new discoveries on metabolic variations in single cells. ${ }^{[11]}$ The highest sensitivity for phosphorylated metabolites was achieved using 9-aminoacridine (9AA) as matrix (Fig. 1). 9AA has very few background peaks, which also facilitates detection of negatively charged species in the low $m / z$ range. The limits of detection for metabolites such as ADP, GDP, ATP, GTP, and acetyl-CoA were found to be between 5 and 12 attomoles using this approach.

There is an on-going trend to carry out treatment of biological samples in microfluidic devices. ${ }^{[12]}$ Moreover, microfluidic platforms are in particular suitable for handling and analyzing single cells. Therefore, a general goal is to develop a microscale interface between the microfluidic sample treatment step and sensitive detection, e.g. by MALDI-MS. The method described above exploited deposition of discrete spots of samples using a piezoelectric microdispenser. ${ }^{[11]}$ However, on-line coupling of microfluidic sample treatment steps with the piezoelectric microdispenser is not possible due to the considerable dead volume of this kind of device. Since high-throughput microfluidic methods normally operate in the continuous flow mode, it would also be very helpful to be able to deposit samples continuously prior to detection by MALDI-MS. Several approaches to deposition have been presented in the scientific and patent literature. ${ }^{[6-10]}$ Examples include deposition on MALDI plates after a separation step such as liquid chromatography ${ }^{[9]}$ or capillary electrophoresis. ${ }^{[6]}$

The objective of the present work was to develop a methodology for continuous deposition of samples on MALDI plates to enable implementation of MALDI-MS in sensitive analysis of samples originating from a microfluidic device. While several commercial systems are available for fraction collection or direct transfer of effluent onto MALDI plates, this kind of discretization of samples in the microscale may lead to loss of resolution and sensitivity of the entire analytical method. This is likely to happen for example due to dilution of very narrow zones of analytes in arbitrarily selected volumes of individual fractions. Although discretization can facilitate analysis of samples deposited as arrays of spots on target plates, MALDI-MS instruments available on the market are capable of high-resolution rastering of the entire plate surface in a process referred to as 'MALDI imaging'. Therefore, it is possible to read continuous traces of samples deposited along MALDI plates following sample treatment in a microfluidic device or a capillary separation column without sacrificing resolution or sensitivity. Continuous deposition of samples driven hy-

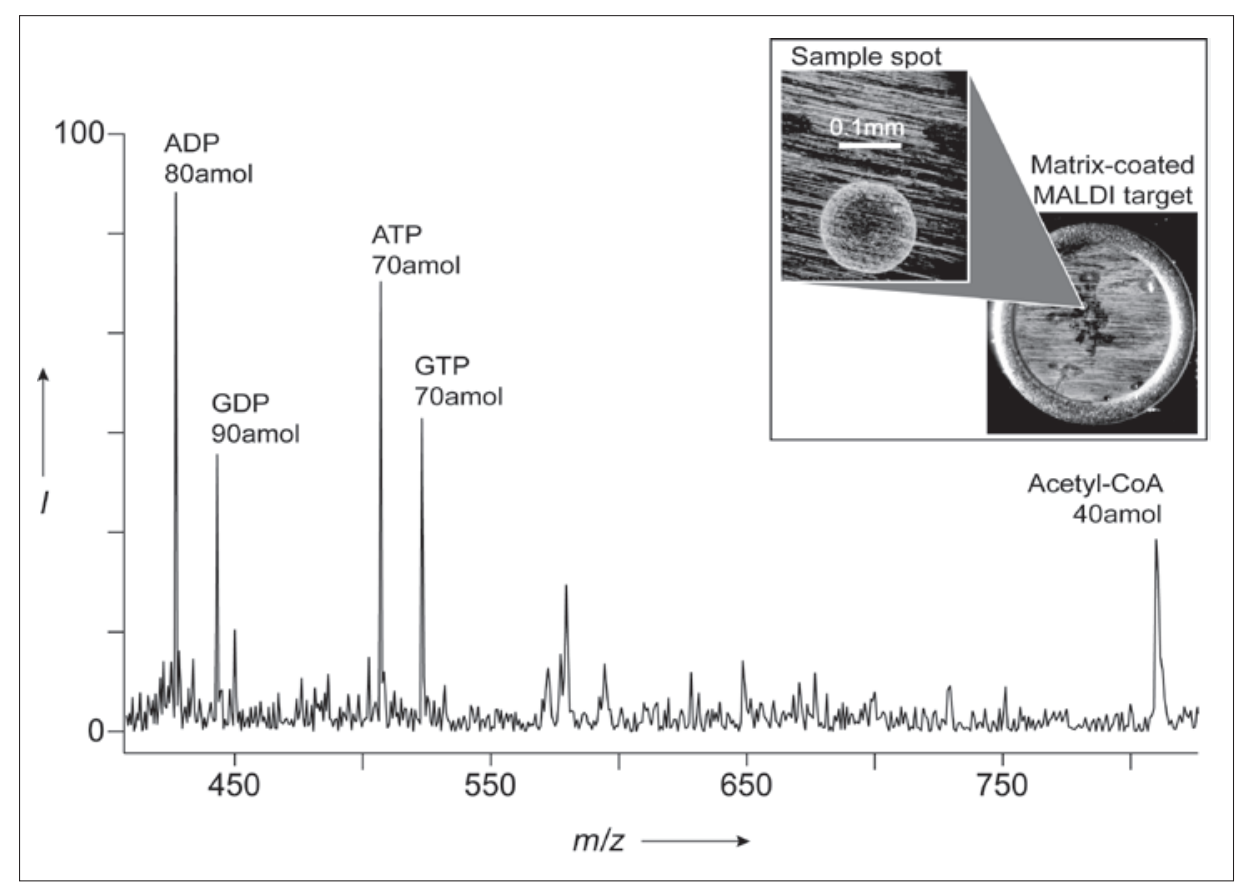

Fig. 1. Single cell sensitivity obtained by MALDI-MS. The samples were spotted onto the surface of target plate using a piezoelectric microdispenser (sample spot shown in the inset). Reprinted from Amantonico et al. ${ }^{[11]}$ Copyright Wiley-VCH Verlag GmbH \& Co. Reproduced with permission.

drodynamically in and out of microfluidic channels directly onto surface of MALDI plates, as described in this article, should also eliminate problems with dead volumes attributed to other deposition techniques, for example those based on piezoelectric effect.

\section{Experimental}

In the deposition method proposed by us, a stainless steel plate is initially coated with a thin layer of matrix. The 9AA matrix is normally deposited using a solution in acetone (up to $\sim 9 \mathrm{mg} \mathrm{ml}^{-1}$ ) or in a mixture of acetone and water. Acetone was especially advantageous due to its fast evaporation at ambient pressure. The coating could be carried out in several ways: i) by fast evaporation of droplets or lanes deposited with a micropipette, ii) by continuous deposition using an XY-stage or XY-plotter, capillary tubing and a syringe pump, iii) by spraying the matrix solution with a sprayer similar to the one used in thin layer chromatography, or iv) by implementing electrospray deposition. By varying the concentration of the matrix as well as by tuning the speed and timing of the deposition process, it was possible to produce layers of different thickness.

Following the coating step, the matrixcoated plate was placed on the top surface of an XY-stage. A polyimide-coated fused silica capillary (in this study: $50 \mu \mathrm{m}$ i.d., $363 \mu \mathrm{m}$ o.d.) can readily be used as a universal output for different microfluidic devices. The output capillary was fixed and positioned right above the target plate, in a way that its outlet was perpendicular to the plate surface. The gap between the capillary tip and the plate was kept as narrow as possible. This distance (Z-dimension relative to the XY-stage) could easily be controlled with a precision translation stage, mounted right above the XY-stage, on which the capillary was mounted (Fig. 2).

At the beginning of the deposition the capillary was moved down, so as to establish the contact of the effluent with the surface of the matrix-coated plate. When a liquid junction was formed between the capillary tip and the plate surface, and stabilized due to the surface tension, the tip was moved away from the plate to maintain a gap of $\sim 100 \mu \mathrm{m}$. Adjustment of this distance helped to minimize the dead volume of the liquid junction, which might contribute to the broadening of sample zones leaving the microfluidic device. It has also been noted that tapering the capillary end as well as surface functionalization to induce hydrophobicity helped to decrease the dead volume of the liquid junction formed between the capillary tip and the plate surface. Due to the continuous movement of XY-stage in a single dimension the deposition of thin bands of samples on the thin layer of matrix was achieved. The wetted area of the matrix surface dried out within seconds. Therefore, one may conclude that for aqueous solutions (as in our study) there are virtually no issues due to diffusion of analytes along the deposition lane. The plate prepared with the method described above was ready to be analyzed 
(A)

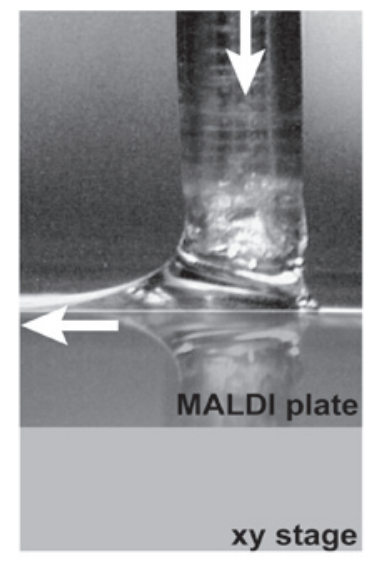

(B)

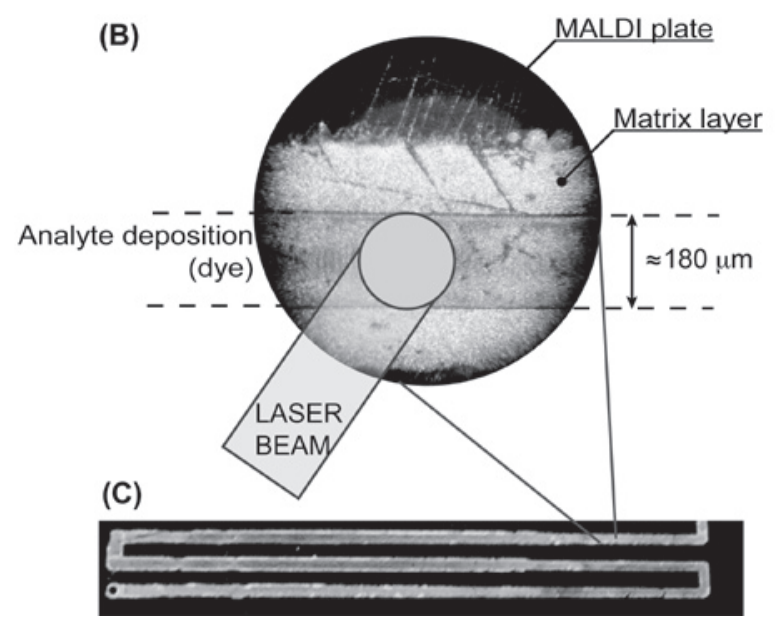

Fig. 2. (A) Deposition of the effluent from a microfluidic device using an $X Y$-stage. White arrows indicate the direction of sample flow inside the capillary and the direction of the target plate movement during the deposition. (B) The resulting trace of effluent (containing rhodamine) on a lane of pre-deposited MALDI matrix (9-aminoacridine). The schematic shows that the diameter of the laser spot (applied during ionization in the MALDI source) is comparable with the width of the sample band obtained during the deposition. (C) 9AA lane on the MALDI plate.

by commercial MALDI-time-of-flight (MALDI-TOF) instruments.

\section{Results and Discussion}

During the optimization of the continuous deposition it was observed that, in order to maintain stable deposition, three critical deposition parameters need to be considered, i.e.: i) sample flow rate, ii) speed of the XY-stage movement, and iii) distance of the capillary tip from the plate. It must be pointed out that these parameters have to be optimized for a certain solvent (due to differences in surface wettability, viscosity and vapor pressure), and their interplay will determine the width of the sample trace deposited on the plate. The width was measured under a microscope following deposition of a red dye, rhodamine, on the thin layer of 9AA matrix (Fig. 2). In our case a band with a width of $\sim 180 \mu \mathrm{m}$ was obtained. This is comparable with the diameter of spots obtained in the previous study, $\sim 160 \mu \mathrm{m}$ (Fig. 1). Such a band width is also comparable with the diameter of the laser spot obtained in the majority of commercial MALDI-TOF systems. In fact, reduction of sample presentation surface on MALDI plates has been shown to increase sampling efficiency in MALDI ionization process. ${ }^{[13]}$ Therefore, minimizing the surface area occupied by the sample may help to improve detection sensitivity necessary to analyze minute amounts of samples normally delivered by microfluidic devices.

The sample trace presented in Fig. 2 was obtained using a flow rate of $250 \mathrm{nl}$ $\mathrm{min}^{-1}$ while the XY-stage was set to move in X-direction with a constant speed of $24 \mathrm{~mm} \mathrm{~min}^{-1}$. Considering these deposi- tion parameters, the volume-per-surface

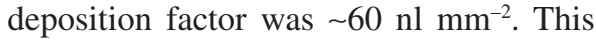
is comparable with the factor estimated for the deposition with the piezoelectric microdispenser presented in our previous study, ${ }^{[11]} \sim 20 \mathrm{nl} \mathrm{mm}^{-2}$ (0.4 $\mathrm{nl}$ droplet deposited onto a spot with a radius of 0.080 $\mathrm{mm}$ and surface area of $0.020 \mathrm{~mm}^{2}$ ). For the method presented above it was also possible to estimate the value of linear deposition efficiency: $\sim 100 \mathrm{nl} \mathrm{cm}{ }^{-1}$. Considering transfer of discrete zones of samples from the microfluidic device onto the plate, this value may help to assess compatibility of the developed deposition method with a microfluidic chip operating at a certain flow rate. By varying the speed of the deposition it is possible to alter the relative widths of the sample zones inside the microfluidic channel (capillary) and the plate. This can also help to avoid losses of resolution of sample zones following the transfer onto the plate.

In a previous study by Chen et al. ${ }^{[9]}$ the matrix was mixed with the column effluent in a Micro-Tee prior to the deposition on the target plate. However, the process of mixing, which involves a highly concentrated matrix solution, may cause problems due to precipitation in the microfluidic channels. In contrast to the previous work, in the present study the matrix was coated on the plate before the deposition, so the effluent could directly be deposited onto the plate. This helped to avoid any problems with precipitation of the matrix and analytes in the microfluidic channel. In addition it can help to avoid further dispersion of analytes in the channel as may occur when mixing the matrix solution with the effluent in a Micro-Tee. It shall also be pointed out that during the deposition ac- cording to the method presented, the capillary tip does not touch the surface of the target plate coated with matrix while the gap between the tip and the plate can precisely be controlled with the Z-dimension translation stage. Precise control of the distance between the capillary tip and the target plate also helps to avoid mechanical scratching of the matrix layer.

We believe that a particular application of the continuous deposition of samples on plates may be in the enhancement of quantitative capabilities of MALDI-MS. The biggest constraint for quantification by MALDI is due to inhomogenity of analyte and matrix distribution within a spot on the MALDI plate. These inhomogeneities are minimized during automated deposition of effluent from a microfluidic device. Another possibility to obtain quantitative data would be to apply a chemometric data treatment; considering the profiles of analyte quantities deposited along the lane as a projection of the concentration profiles along the microfluidic channel, and assuming a defined (e.g. Gaussian) distribution of analytes within a sample zone dispersed in the microfluidic channel.

In some studies it was demonstrated that, for particularly large cells such as neurons, single cell analysis can be carried out with MALDI just by depositing an intact cell on the MALDI plate. ${ }^{[14]}$ However, in most cases, single cell sample treatment including lysis and purification/separation is indispensable. ${ }^{[15]}$ While the MS itself can be fully automated, a considerable amount of manual labor is required to prepare the samples for MS analysis. This can be simplified by implementing one of the existing microfluidic platforms. ${ }^{[12,15-20]}$ Therefore in the final version of our platform, following cell lysis, the analytes and cell debris will be separated and the complete metabolome transferred onto a MALDI plate as described above (Fig. 3).

\section{Concluding Remarks and Future Trends}

The continuous sample deposition method described here is ideal for coupling microfluidics with laser desorption/ ionization (LDI) mass spectrometry since it is compatible with the amounts and volumes of samples typically delivered by microfluidic devices. The deposition protocol described here should also be compatible with any microfluidic platform, including lab-on-a-chip, lab-on-capillary as well as nano-liquid chromatography and capillary electrophoresis. It should be pointed out that the deposition technique presented may in fact readily be applied in conjunction with matrix-free LDI methods, for example desorption ionization on silicon 


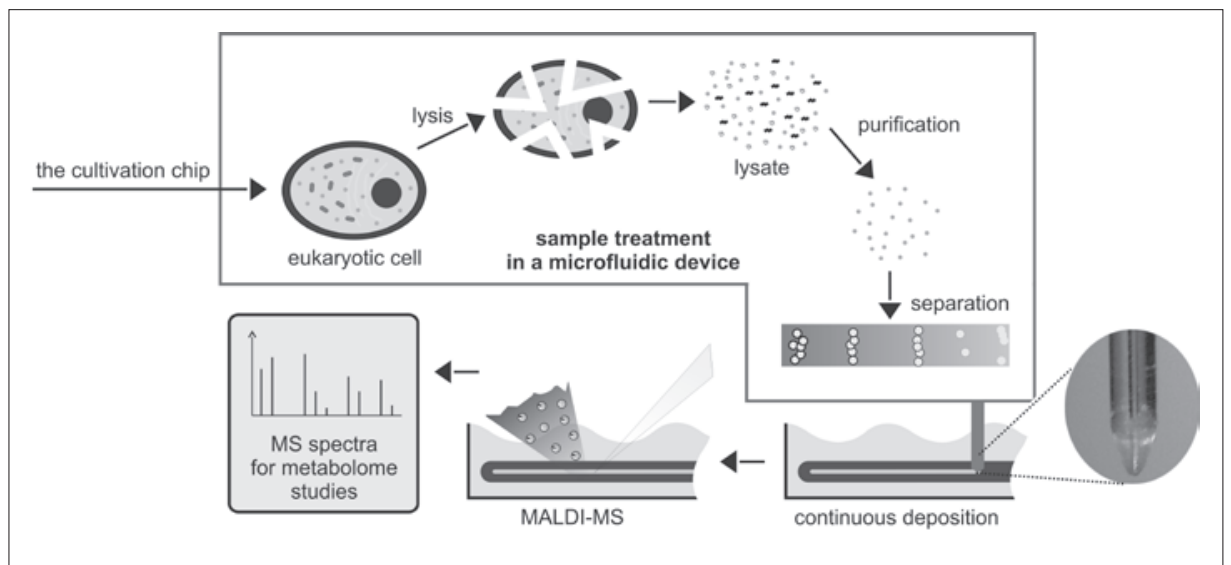

Fig. 3. Schematic of a microTotal Analysis System for the envisioned single cell analysis platform incorporating continuous deposition methodology described in the text. The inset shows a tapered capillary tip.

(DIOS) or nanostructure initiator mass spectrometry (NIMS); ${ }^{[3]}$ as well as surface analysis techniques. Further strategies for increasing detection sensitivity, such as minimizing the surface area occupied by the band of effluent deposited on the MALDI plates, are currently being investigated.

\section{Acknowledgements}

We would like to thank Dr Matthias Heinemann and Dr Nils Goedecke for helpful discussions and contributions to the metabolomics and microfluidics parts, respectively. P.L.U. would like to acknowledge the European Union for an individual postdoctoral intra-European Marie Curie fellowship and the related research funding (PIEF-GA-2008-219222 - MESEL).

Received: February 2, 2009

[1] S. G. Villas-Boas, S. Mas, M. Akesson, J. Smedsgaard, J. Nielsen, Mass Spectrom. Rev. 2005, 24, 613 .

[2] 'The Handbook of Metabonomics and Metabolomics', Ed. J. C. Lindon, J. K. Nicholson, E. Holmes, 2007, Elsevier BV, Amsterdam.

[3] N. Blow, Nature 2008, 455, 697.

[4] J. Preisler, F. Foret, B. L. Karger, Anal. Chem. 1998, 70, 5278 .
[5] H. Orsnes, R. Zenobi, Chem. Soc. Rev. 2001, 30, 104.

[6] C. W. Huck, R. Bakry, L. A. Huber, G. K. Bonn, Electrophoresis 2006, 27, 2063.

[7] A. Brock, C. M. Shaw, R. C. Downs, Patent Publication No.WO 02/062475 A1, 2002.

[8] C. Stacey, Patent Publication No. US2004/0023410 A1, 2004.

[9] H. Chen, T. Rejtar, V. Andreev, E. Moskovets, B. L. Karger, Anal. Chem. 2005, 77, 2323.

[10] V. C. Chen, H. Perreault, Patent Publication No. CA 2551362, 2006.

[11] A. Amantonico, J. Y. Oh, J. Sobek, M. Heinemann, R. Zenobi, Angew. Chem., Int. Ed. 2008, 47, 5382.

[12] K. Ohno, K. Tachikawa, A. Manz, Electrophoresis 2008, 29, 4443.

[13] L. Li, R. E. Golding, R. M. Whittal, J. Am. Chem. Soc. 1996, 118, 11662.

[14] R. L. Dahlgren, J. S. Page, J. V. Sweedler, Anal. Chim. Acta 1999, 400, 13.

[15] L. M. Borland, S. Kottegoda, K. S. Phillips, N. L. Allbritton, Annu. Rev. Anal. Chem. 2008, 1, 191.

[16] J. Gao, X. F. Yin, Z. L. Fang, Lab Chip 2004, 4, 47.

[17] H. Wu, A. Wheeler, R. N. Zare, Proc. Natl. Acad. Sci. USA 2004, 101, 12809.

[18] D. Di Carlo, L. P. Lee, Anal. Chem. 2006, 78, 7918.

[19] J. El-Ali, P. K. Sorger, K. F. Jensen, Nature 2006, 442, 403

[20] W. H. Huang, F. Ai, Z. L. Wang, J. K. Cheng, J. Chromatogr. B 2008, 866, 104. 